\title{
Molecularly imprinted hydrogels for application in aqueous environment
}

\author{
Anna Korytkowska-Wałach
}

Received: 26 October 2011/Accepted: 30 September 2012/Published online: 7 October 2012

(C) The Author(s) 2012. This article is published with open access at Springerlink.com

\begin{abstract}
Molecularly imprinted thermoresponsive hydrogels for dopamine recognition were synthesized using $N$-isopropylacrylamide as the temperature-sensitive element, methacrylic acid or itaconic acid as functional monomer and $N, N$ '-methylenebisacrylamide or ethylene glycol dimethacrylate as crosslinking agent. The stoichiometry of the template-functional monomer prepolymerization complexes was determined by ${ }^{1} \mathrm{H}$ NMR experiments according to the Job method of continuous variation as well as signal line width analysis. It was found that dopamine hydrochloride forms complexes with methacrylic acid and itaconic acid with 1:3 and 2:3 template to functional monomer stoichiometry, respectively. Hydrogels composed of itaconic acid and with appropriate template content revealed the highest efficiency of imprinting process and relatively high selectivity of dopamine hydrochloride rebinding in water near the phase transition temperature.
\end{abstract}

Keywords Molecular imprinting · Prepolymerization complex · Dopamine · Hydrogels · Thermosensitive polymers

\section{Introduction}

Molecular imprinting (MI) into organic polymers, first reported by Wulff and Sarhan [1], nowadays is widely reported technique of preparing polymeric materials with molecular recognition elements. Those kinds of materials can find applications as separating agents, elements in sensors, catalysts, etc. The first step of polymerization via MI technique is complex formation between monomers and printed molecules,

\footnotetext{
A. Korytkowska-Wałach $(\bowtie)$

Department of Organic Chemistry, Bioorganic Chemistry and Biotechnology, Silesian University of Technology, Krzywoustego 4, 44-100 Gliwice, Poland e-mail: anna.walach@polsl.pl
} 
termed as templates. Complexes formed spontaneously in the porogen are next sterically fixed during crosslinking polymerization. The crosslink density is determined by the content of crosslinking agent. After removing the imprinted molecules from polymer matrix, artificial recognition sites are created.

Monomer-template complexes can be formed either by strong covalent interactions or weak non-covalent ones (e.g. hydrophobic interactions, ionic and hydrogen bonding). The second way is often used due to the simplicity of the method. Non-covalent imprinting can be performed easily by mixing components to form prepolymerization complexes. Removing of template from polymerized system is followed simply through extraction. Selection of appropriate monomers and porogen (solvent in which complexes are formed) seems to be crucial in noncovalent imprinting. Additional difficulty of this method inheres in proper monomer-template ratio fitting. Too high a monomer-template ratio yields polymer with non-specific binding, whereas too low one leads to insufficient template complexation and low amount of sites with specific shape and functionality [2].

Most of the investigated and used MI materials work in organic, non-polar solvents due to limited interactions between template or monomer with the solvent. On the other hand, most of the important biological substances are water soluble and there is a need of their determination directly from biological fluids (plasma, urine or saliva). Some attempts of adaptation by hydrophobization of molecularly imprinted polymers for binding molecules in aqueous environment were done by few researching groups. Piletska et al. proposed treatment of MI polymer with mineral oil in order to create a hydrophobic shield for protecting functional groups [3]. Some authors used polymerizable $\beta$-cyklodextrine as a hydrophobic moiety [4] or artificial receptors that provided a preorganized hydrophobic environment [5].

In this study, for recognizing from aqueous environment, concept of use of thermosensitive polymers was utilized, where effectiveness of binding molecules can be enhanced at phase transition temperature by decreasing the relative distance of the functional groups inside the imprinted cavities [6]. There were several reports recently focused on the development of temperature sensitive molecularly imprinted polymers. Suedee et al. used thermosensitive acrylamide hydrogel for extraction of adregenic drugs from urine [7]. 4-Aminopiridine and L-pyroglutamic acid were imprinted as a model analyte into thermosensitive poly $(N$-isopropylacrylamide $)$ gels $[8,9]$. The authors indicated that these hydrogels showed selectivity for used templates above critical temperature.

In this research, thermoresponsive hydrogels based on $\mathrm{N}$-isopropylacrylamide (NIPAm) and methacrylic acid (MAc) or itaconic acid (ItAc) were used. Dopamine hydrochloride having catechol and amine groups was chosen as a binding molecule. Determination of some catecholamines as well as their metabolites from biological fluids is important in the diagnosis of catecholamine-secreting tumours such as pheochromocytomas, paragangliomas and neuroblastomas [10]. Dopamineimprinted polymers have been studied earlier by the Sugimoto [11], Suedee [7] and Luliński [12] groups, where $\mathrm{N}$-isopropylacrylamide-acrylic acid and acrylamide-methacrylic acid thermosensitive hydrogels, or not thermosensitive ones based on methacrylic acid, were tested, respectively. 


\section{Experimental}

\section{Materials}

All chemicals and solvents were of commercial grade and used as received.

$N$-isopropylacrylamide (NIPAm), N,N'-methylenebisacrylamide (BIS), methacrylic acid (MAc), itaconic acid (ItAc), 2,2'-azobis(isobutyronitrile) (AIBN), dopamine hydrochloride, catechol, tyramine and deuterated dimethyl sulfoxide (DMSO- $\mathrm{d}_{6}$ ) were obtained from Acros Organics (Chemiatrade, Gliwice, Poland). Ethylene glycol dimethacrylate (EGDMA), L-3,4-dihydroxyphenylalanine (L-DOPA) were purchased from Sigma-Aldrich Co. (Poznań, Poland). Dimethyl sulfoxide (DMSO), methyl alcohol and acetic acid were obtained from POCh (Gliwice, Poland).

Instruments

${ }^{1} \mathrm{H}$ NMR spectra were recorded at ambient temperature with the aid of UNITY/ INOVA 300 or $600 \mathrm{MHz}$ spectrometers (Varian) using DMSO- $\mathrm{d}_{6}$ as a solvent and TMS as an internal reference.

UV-VIS wavescans and photometry measurements were done using a Hitachi double beam spectrophotometer Series U-2910.

\section{Hydrogel synthesis}

Molecularly imprinted hydrogels (MIP) were obtained by free radical crosslinking copolymerization. Prior to polymerization, appropriate amount of monomers, initiator and template were dissolved in porogen. Next, resulted solutions (17 wt\% in DMSO) were purged with nitrogen for $15 \mathrm{~min}$ and transferred to polymerization forms - two sealed glass plates, which were then placed in a drying oven at $65{ }^{\circ} \mathrm{C}$ for $24 \mathrm{~h}$. The detailed feed compositions and sample codes of the hydrogels are given in Table 1. After polymerization, resulted hydrogels were soaked in distilled water for 1 day. Then some discs with $5 \mathrm{~mm}$ diameter were cut. The remainder of the gels were crushed. Hydrogel samples in the form of discs as well as ground ones where treated with hot water (for $2 \mathrm{~h}$ ) and then placed in Soxhlet apparatus. Methanol/acetic acid solution in 2:1 v/v ratio (for $24 \mathrm{~h}$ ) and subsequently methanol (for $12 \mathrm{~h}$ ) were used for extraction of template from the gels.

The same preparation procedure was used for non-imprinted hydrogels (I_NMIP, II_NMIP, 1_NMIP, 2_NMIP, 3_NMIP, 4_NMIP, 5_NMIP, 6_NMIP) as a control, i.e. without addition of the template. Extraction step with methanol/acetic acid solution was also omitted for these hydrogels.

Performed analyses

\section{Stoichiometry of the prepolymerization complex determination}

The stoichiometry of the dopamine-methacrylic acid and dopamine-itaconic acid complexes was determined according to the Job's method of continuous variations 
Table 1 The feed compositions and sample codes of the imprinted hydrogels

\begin{tabular}{|c|c|c|c|c|c|c|c|c|c|}
\hline \multirow[t]{2}{*}{ Composition } & \multicolumn{9}{|c|}{ Sample code } \\
\hline & I_MIP & II_MIP & 1_MIP & 2_MIP & 3_MIP & 4_MIP & 5_MIP & $5^{\prime} \_$MIP & 6_MIP \\
\hline NIPAm (g) & 0.5 & 0.5 & 0.5 & 0.5 & 0.5 & 0.5 & 0.5 & 0.5 & 0.5 \\
\hline $\operatorname{MAc}(\mathrm{wt} \%)^{\mathrm{a}}$ & 25 & 20 & & & & & & & \\
\hline ItAc $(\mathrm{wt} \%)^{\mathrm{a}}$ & & & 10 & 10 & 10 & 10 & 10 & 10 & 10 \\
\hline EGDMA $(w t \%)^{\mathrm{b}}$ & & & & 12.4 & & 17.5 & & & 25.7 \\
\hline BIS $(w t \%)^{b}$ & 10 & 10 & 10 & & 15 & & 20 & 20 & \\
\hline Dopamine $\cdot \mathrm{HCl}^{\mathrm{c}}$ & $1: 3$ & $1: 3$ & $2: 3$ & $2: 3$ & $2: 3$ & $2: 3$ & $2: 3$ & $1: 6$ & $2: 3$ \\
\hline
\end{tabular}

Based on ${ }^{\mathrm{a}}$ NIPAm and ${ }^{\mathrm{b}}$ total amount of monomers

c Template to functional monomer (MAc or ItAc) molar ratio

by the ${ }^{1} \mathrm{H}$ NMR analysis in deuterated DMSO. Equimolar solutions $(0.04 \mathrm{M})$ of carboxylic acid and dopamine hydrochloride in $\mathrm{DMSO}-\mathrm{d}_{6}$ were prepared, respectively, dried over molecular sieves $(4 \AA)$ and distributed among 11 NMR tubes in such a way that the ratios of monomer and templates varied between 0:1 and 1:0 (with a constant sample volume of $0.7 \mathrm{~mL}$ ). NMR line width analyse were done simultaneously. Experiments were carried out in triplicate. Experimental data were related to the dopamine hydrochloride molar fraction calculated from NMR spectra.

Characterization of the swelling properties of the gels

In order to determine the swelling ratio $(S)$ of non-imprinted hydrogels, the dried samples were immersed in water at specific temperature until equilibrium was confirmed (usually $48 \mathrm{~h}$ ). After that, the samples were removed from the water, wiped with filter paper and weighted. Swelling ratio at given temperature was calculated according to the equation:

$$
S=\frac{w_{\mathrm{s}}-w_{\mathrm{d}}}{w_{\mathrm{d}}} \cdot 100(\%)
$$

where $w_{\mathrm{s}}$ and $w_{\mathrm{d}}$ are the hydrogels weights in the swollen and dry state, respectively.

\section{Dopamine hydrochloride binding test}

Binding of the template molecule tests were performed using UV-VIS spectrophotometry. Briefly, $0.01-0.02 \mathrm{~g}$ of ground dry polymer was placed in $6 \mathrm{ml}$ of aqueous solutions of dopamine hydrochloride $(50 \mu \mathrm{g} / \mathrm{mL})$ and kept at given temperature for $48 \mathrm{~h}$. Then the polymer was filtered off, and the concentration of dopamine hydrochloride in the filtrate was measured at $\lambda=279 \mathrm{~nm}$ using calibration curve. All experiments were performed in triplicate, and data were expressed as the mean \pm standard deviation.

\section{Selectivity test}

The selectivity evaluation was examined similar to binding experiments. Solutions $(50 \mu \mathrm{g} / \mathrm{mL})$ of catechol, L-DOPA and tyramine were subjected, respectively, to 
competitive binding assay. Concentration changes were measured using calibrations curves (analytical wave length for catechol and tyramine was $274 \mathrm{~nm}$ and for LDOPA $279 \mathrm{~nm}$ ). All experiments were performed at given temperature in triplicate, and data were expressed as the mean \pm standard deviation.

\section{Results and discussion}

NMR prepolymerization complex analyses

In molecularly imprinted polymers with high-molecular recognition properties, the strength and stoichiometry of the monomer-template interactions are of great importance. Modelling of molecular interactions of the templates with functional monomers is sometimes studied in order to preselect the functional monomers used in the synthesis of MIP. Theoretical analysis of the intermolecular interactions in the prepolymerization complexes between dopamine hydrochloride and functional monomers in different solvent was reported by Luliński et al. [12]. Investigations of template-functional monomer complexation by ${ }^{1} \mathrm{H}$ NMR are also informative and give insights at stoichiometry and association strength of the prepolymerization complexes. Suedee et al. tested methacrylic acid and acrylamide for their ability to generate specific binding sites for the dopamine molecule in DMSO- $\mathrm{d}_{6}$ by ${ }^{1} \mathrm{H}$ NMR titration [13]. The determined apparent dissociation constant of the complex between dopamine and functional monomers suggests formation of rather strong complex in polar aprotic solvent. Authors postulated complex with 1:1 stoichiometry with interaction between the carboxylic group of MAc and hydroxyl group of dopamine. They did not identify by spectroscopic analysis hydrogen bond interactions with the primary amino group of the template which were predicted by HyperChem. That was explained by existence of the electrostatic interactions or weak hydrogen bond between $\mathrm{NH}_{3}{ }^{+}$of the template and the carboxylic group of MAc in aprotic solvent. In this study, ${ }^{1} \mathrm{H}$ NMR analyses of the prepolymerization complexes formed between methacrylic acid or itaconic acid and dopamine hydrochloride (MAc-D and ItAc-D) in deuterated DMSO were performed. In contrast to Suedee group, the line widths of labile proton signals were examined. Broadening of the signals could be expected due to a slow exchange of equivalent protons in the complexes $[14,15]$. By plotting the peak width versus molar ratio of one of the constituents the complexes stoichiometry could be find.

In the methacrylic acid and dopamine hydrochloride mixtures for low-molar fraction of dopamine, exchangeable protons of the ammonium and two hydroxyl groups give one broadened signal at $\delta=7.0-9.5 \mathrm{ppm}$ in ${ }^{1} \mathrm{H}$ NMR spectra (Fig. 1a). Proton of carboxylic group gives second very broad signal at $\delta=9.5-13 \mathrm{ppm}$. For higher content of the dopamine hydrochloride in the prepolymerization mixture protons of the ammonium and hydroxyl groups gives two separate signals.

Considerable broadness of all aforementioned signals indicates that strong interaction with contribution of those protons takes place. Analysing ${ }^{1} \mathrm{H}$ NMR spectra of itaconic acid and dopamine presented at Fig. 1b, beginning from low content of the template $\left(x_{\mathrm{D}}=0.1\right)$, there are four visible signals separately: signal 

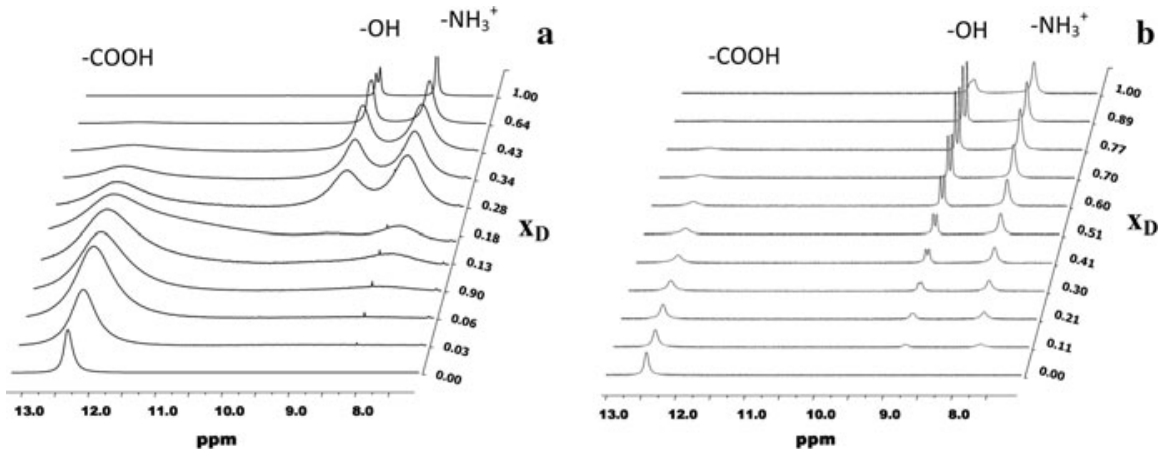

Fig. $1{ }^{1} \mathrm{H}$ NMR spectra of the heteroatomic protons in the dopamine-methacrylic acid (a) and dopamine-itaconic acid (b) mixtures with different dopamine molar fraction $\left(x_{\mathrm{D}}=0-1\right)$

of the ammonium protons $(\delta=7.7-8.1 \mathrm{ppm})$, two signals of two hydroxyl of the catechol group $(\delta=8.8-8.9 \mathrm{ppm})$ and signal of the carboxylic groups $(\delta=12.3-12.7 \mathrm{ppm})$. Figure 2 depicts line width at half height of the carboxylic protons signal versus molar fraction of the dopamine in the mixture with methacrylic and itaconic acid, respectively.

In the case of the methacrylic acid, two maxima are observed. The first one for $x_{\mathrm{D}}=0.2-0.3$ could correspond to the formation of the $\mathrm{MAc}_{3} \mathrm{D}$ complex, where dopamine hydrochloride molecule interacts with methacrylic acid through three binding sites. The second maximum observed for $x_{\mathrm{D}}=0.3-0.5$ corresponds to the formation of the two methacrylic acid-dopamine complexes with the 1:1 and 2:1 stoichiometry. In the case of methacrylic acid-dopamine pair observation of the chemical shift changes during complexation was difficult due to significant line broadening. For itaconic acid and dopamine mixtures line width as well as chemical shift analyses were conducted. Observation of spectral line width of carboxylic proton of the itaconic acid for different dopamine molar fraction, indicated formation of the 1:2, 3:2 and 5:2 itaconic acid-dopamine complexes. Postulated structure for the $\mathrm{ItAcD}_{2}$ complex assumed the most probable electrostatic interaction between ammonium and carboxyl groups. During $\operatorname{It}_{\mathrm{C}_{3}} \mathrm{D}_{2} \operatorname{complex}$ formation probably electrostatic interactions between carboxylic group and basic nitrogen with additional hydrogen bond between hydroxyl group and carboxylic carbonyl group takes place. In the case of engaging one of the aromatic hydroxyl group, ItAc $\mathrm{D}_{2}$ complex could be formed. The third maximum suggests that the 5:2 and probably higher order template-monomer complexes are created.

The signals of the protons of ammonium group and one of the hydroxyl groups of dopamine experience downfield shift as the dopamine fraction increases. The Job plot of interactions indicates 3:2 monomer-template stoichiometry (Fig. 3), suggesting that this complex is the most stable and dominant one.

Hydrogels characterization

Synthesized non-imprinted (NMI) hydrogels have a so-called lower gel transition temperature (LGTT) [16] and show thermally induced, reversible changes in their 

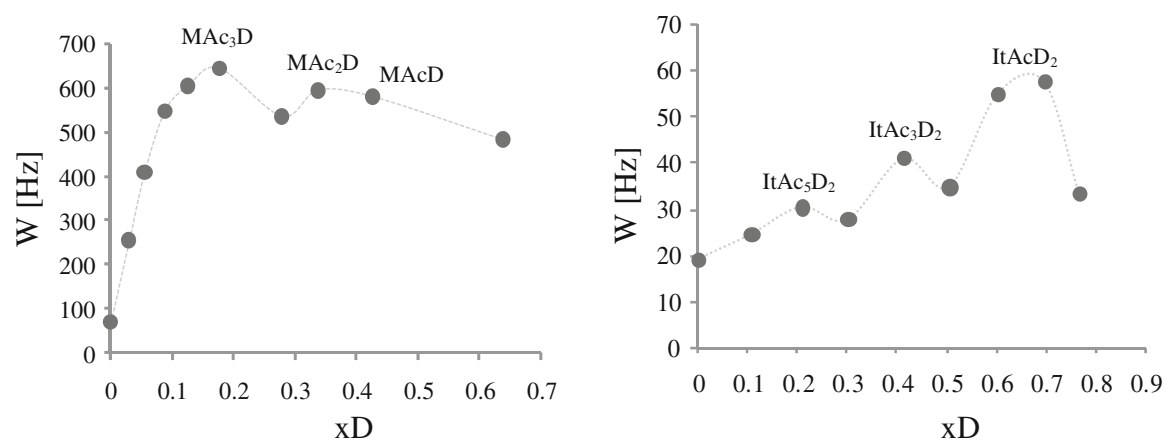

Fig. 2 Signal line width (measured at half height of signal) for carboxylic protons of methacrylic acid (left) and itaconic acid (right) versus molar fraction of the dopamine (both referenced to TMS signal line width)
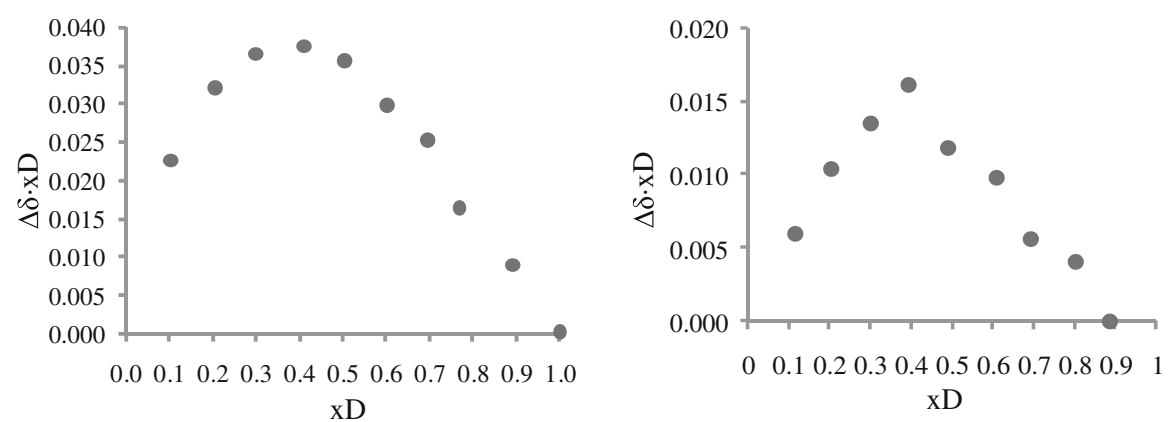

Fig. 3 Job's plots for the interaction between dopamine and itaconic acid in DMSO by monitoring chemical shifts of the ammonium protons signal (left) and hydroxyl protons signal of the dopamine (right)

swelling behaviour. Hydrogels with methacrylic acid show phase transition at $22-32{ }^{\circ} \mathrm{C}$ while hydrogels composed with itaconic acid at $30-40{ }^{\circ} \mathrm{C}$ temperature range (Fig. 4). One can observe an influence of the kind of crosslinker and crosslink density on the swelling properties but not on the LGTT value.

Analogical hydrogels were synthesized with dopamine hydrochloride used as the print molecule with stoichiometry determined from NMR experiments, i.e. 1:3 dopamine to methacrylic acid and 2:3 template to itaconic acid molar ratio. Additionally, 5'_MIP hydrogel with an fourfold excess of itaconic acid was synthesized in order to shift eventually the equilibrium of binding to the associated complex. All non-imprinted as well as imprinted hydrogels were tested towards determination of the optimal temperature for binding dopamine. Figure 5 presents an influence of temperature on dopamine binding property by the exemplary MI and NMI hydrogels.

As it is seen, the highest content of bonded dopamine was reached at 20 to ca. $40{ }^{\circ} \mathrm{C}$ temperature range with the maximum near the LGTT. The effectiveness of binding dopamine decreased above LGTT due to more compact polymer network which hinder the entrance for molecules. The effectiveness of rebinding template as 

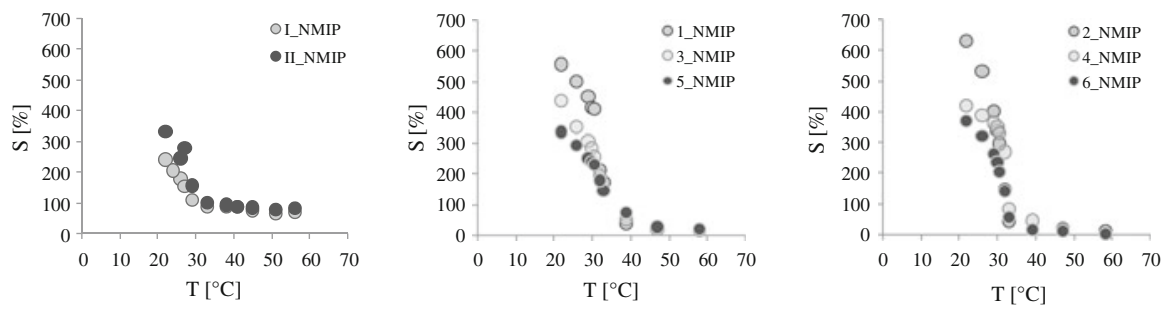

Fig. 4 Swelling ratio of non-imprinted hydrogels as a function of temperature
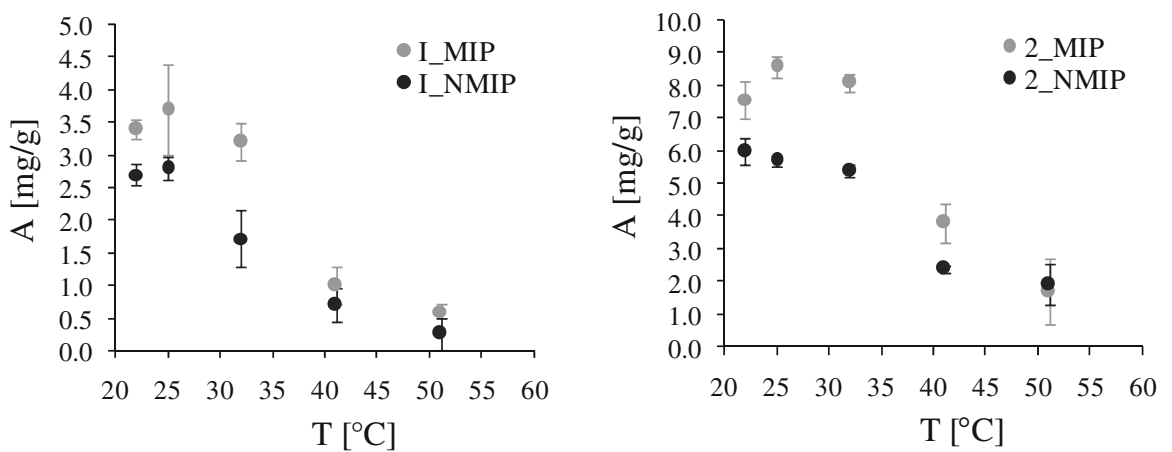

Fig. 5 Dopamine binding by I_MIP, I_NMIP, 2_MIP and 2_NMIP at different sets of temperature

well as selectivity was determined at temperature at which LGTT phenomenon occurs (Fig. 6).

Effectiveness of binding of the dopamine, i.e. amount of $\mathrm{mg}$ of template bound per $1 \mathrm{~g}$ of polymer, is much smaller in the case of methacrylic acid than itaconic acid based hydrogels. Possibly reason for this could lie in strong tendency of MAc to form hydrogen-bonded dimers [17]. In the case of dimerization of methacrylic acid, interaction with the template molecule will be precluded. When dimerization occurs increasing content of the functional monomer in hydrogels should not result in significant enhancement of binding dopamine effectiveness. As it is seen from Fig. 6 amount of bound template molecule in rebinding process is independent on methacrylic acid content in the hydrogels.

Amounts of bound dopamine are ca. fourfold greater in the case of itaconic-based hydrogels, and influence of the crosslink density as well kind of crosslinking agent on the effectiveness of binding are negligible.

Efficiency of imprinting process could be expressed by means ratio of dopamine amount bound by the imprinted hydrogels to the amount of dopamine bound by the control non-imprinted ones $\left(A_{\mathrm{MIP}} / A_{\mathrm{NMIP}}\right)$ (Fig. 7).

Uppermost $\mathrm{A}_{\mathrm{MIP}} / \mathrm{A}_{\mathrm{NMIP}}$ value was demonstrated by hydrogels based on methacrylic acid (I_MIP, II_MIP), and those hydrogels with itaconic acid which were crosslinked with $20 \mathrm{wt} \%$ N,N'-methylenebisacrylamide (BIS) (5_MIP and $5^{\prime} \_$MIP). When analysing experimental data collected for lightly crosslinked 


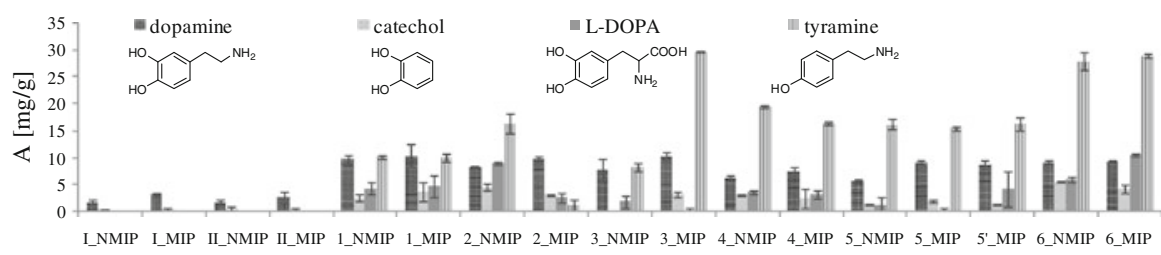

Fig. 6 Binding of the dopamine hydrochloride and selected similar chemical molecules by the hydrogels at LGTT

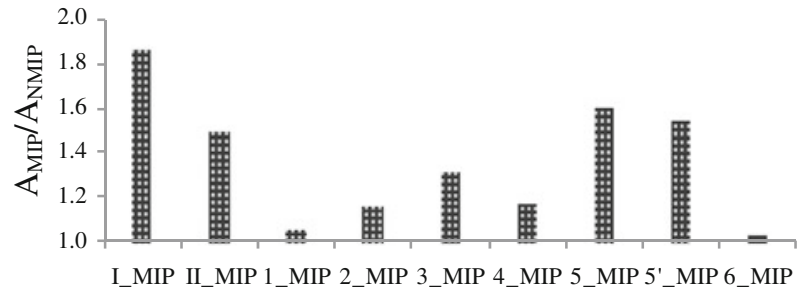

Fig. 7 Efficiency of imprinting dopamine of imprinted hydrogels in relation to their non-imprinted analogues at LGTT

polymers with itaconic acid acting as functional monomer, none of the essential differences in binding properties between imprinted and related non-imprinted hydrogels are observed. Probably, the relatively low content of crosslinking agents enable movement of polymers chains, so the size and shape of binding cavity can be easily changed, which in turn entailed non-specific binding.

The hydrogels containing more rigid and hydrophobic molecule of the ethylene glycol dimethacrylate (EGDMA) as a crosslinker were expected to demonstrate lower swelling degree than hydrogels crosslinked with $N, N$ '-methylenebisacrylamide. It is known that BIS provides high flexibility and conformational adaptability to crosslinked polymers [13]. Meanwhile the swelling properties of hydrogels presented on Fig. 5 indicate that those hydrogels possess a slightly lower value of swelling degree than EGDMA crosslinked ones.

Regarding enhancement of dopamine imprinting process for the higher crosslinked hydrogels, more advantageous was to use BIS than EGDMA. An explanation of both decreased swelling and enhanced dopamine binding could be that BIS molecules participate in more extent that EGDMA molecules in intramolecular hydrogen bonds which act as additional physical crosslinks increasing the effective crosslink density and additionally stabilizing shape of binding cavity.

Effectiveness of catechol molecule binding was examined for all hydrogels. In the case of hydrogels with itaconic acid selectivity was tested on L-3,4dihydroxyphenylalanine (L-DOPA) and tyramine additionally.

Performed catechol and L-DOPA binding tests showed relatively high selectivity in regard to those molecules. Hydrogels with equal or higher than $15 \mathrm{wt} \%$ crosslinking agent content demonstrate high tendency for binding tyramine molecule. Tyramine (4-(2-aminoethyl)phenol) in contrast to dopamine molecule 
(4-(2-aminoethyl)benzene-1,2-diol) is not substituted by second hydroxyl group, and it was rather suspected that tyramine would posses high-binding affinity to the binding sites created by dopamine in hydrogels, especially that hydroxyl group at second position of the dopamine probably does not participate in binding template to functional monomer process, because no change in chemical shift was observed for proton signal of this hydroxyl group. The similar conclusions were found by Luliński group on the basis of modelling interactions by the HyperChem program in dopamine cation with methacrylic acid or acrylonitryle complexes [12].

Reduced template to functional monomers molar ratio did not bring enhanced effectiveness and selectivity in dopamine rebinding process.

\section{Conclusion}

In this study, stoichiometry in the prepolymerization complexes of the dopamine with methacrylic or itaconic acid was determined by simple ${ }^{1} \mathrm{H}$ NMR observations in order to ensure the most optimal distribution and arrangement of the binding sites in the synthesized thermoresponsive MIPs. Effectiveness of binding dopamine hydrochloride by those hydrogels is higher near the phase transition temperature at which the access of bound molecule into polymer matrix is still possible and the distance between functional groups contracted enabling effective interactions of imprinted molecule with recognition sites. Amount of bound dopamine is not influenced by the degree of crosslinking density as well as by the kind of crosslinking agent. Kind of used carboxylic acid as functional monomer exhibits essential impact on the binding properties. Hydrogels with itaconic acid are four times more effective in binding dopamine than corresponding methacrylic acidbased hydrogels. The best efficiency of imprinting dopamine (efficiency in relation to non-imprinted analogues) has been found for methacrylic acid containing hydrogels and those with itaconic acid crosslinked with $20 \mathrm{wt} \%$ BIS. Synthesized hydrogels show good selectivity with respect to catechol and L-DOPA molecules, whereas tyramine is preferably bound.

Regarding selectivity and both the best effectiveness in binding dopamine and efficiency of imprinting dopamine process, hydrogels composed of itaconic acid, highly crosslinked with $N, N$ '-methylenebisacrylamide, are the most promising materials for dopamine recognition in aqueous environments.

Open Access This article is distributed under the terms of the Creative Commons Attribution License which permits any use, distribution, and reproduction in any medium, provided the original author(s) and the source are credited.

\section{References}

1. Wulff G, Sarhan A (1972) The use of polymers with enzyme-analogous structures for the resolution of racemates. Angew Chem Int Ed Engl 11:341-342

2. Li W, Li S (2007) Molecular imprinting: a versatile tool for separation, sensors and catalysis. Adv Polym Sci 206:91-210 
3. Piletska EV, Romero-Guerra M, Guerreiro AR, Karim K, Turner APF, Piletsky SA (2005) Adaptation of the molecular imprinted polymers towards polar environment. Anal Chim Acta 542:47-51

4. Asanuma H, Akiyama T, Kajiya K, Hishiya T, Komiyama M (2001) Molecular imprinting of cyclodextrin in water for the recognition of nanometer-scaled guests. Anal Chim Acta 435:25-33

5. Kim J, Raman B, Ahn KH (2006) Artificial receptors that provides a preorganized hydrophobic environment: a biomimetic approach to dopamine recognition in water. J Org Chem 71:38-45

6. Alvarez-Lorenzo C, Concheiro A (2004) Molecularly imprinted polymers for drug delivery. J Chromatogr B 804:231-245

7. Suedee R, Seechamnanturakit V, Canyuk B, Ovatlarnporn C, Martin GP (2006) Temperature sensitive dopamine-imprinted (N, N-methylene-bis-acrylamide cross-linked) polymer and its potential application to the selective extraction of adrenergic drugs from urine. J Chromatogr A 1114:239-249

8. Liu XY, Ding XB, Guan Y, Peng YX, Long XP, Wang XC, Chang K, Zhang Y (2004) Fabrication of temperature-sensitive imprinted polymer hydrogel. Macromol Biosci 4:412-415

9. Liu XY, Guan Y, Ding XB, Peng YX, Long XP, Wang XC, Chang K (2004) Design of temperature sensitive imprinted polymer hydrogels based on multiple-point hydrogen bonding. Macromol Biosci 4:680-684

10. Simon T, Hero B, Hunneman DH, Berthold F (2003) Tumor markers are poor predictors for relapse or progression in neuroblastoma. Eur J Cancer 39:1899-1903

11. Matsui J, Akamatsu K, Hara N, Miyoshi D, Nawafune H, Tamaki K, Sugimoto N (2005) SPR sensor chip for detection of small molecules using molecularly imprinted polymer with embedded gold nanoparticles. Anal Chem 77:4282-4285

12. Luliński P, Maciejewska D, Bamburowicz-Klimkowska M, Szutowski M (2007) Dopamineimprinted polymers: template-monomer interactions, analysis of template removal and application to solid phase extraction. Molecules 12:2434-2449

13. Suedee R, Seechamnanturakit V, Suksuwan A, Canyuk B (2008) Recognition properties and competitive assays of dual dopamine/serotonin selective molecularly imprinted polymer. Int J Mol Sci 9:2333-2356

14. Sellergen B, Lepistö M, Mosbach KJ (1988) Highly enantioselective and substrate-selective polymers obtained by molecular imprinting utilizing noncovalent interactions. NMR and chromatographic studies on the nature of recognition. J Am Chem Soc 110:5853-5860

15. O'Mahony J, Molinelli A, Nolan K, Smyth MR, Mizaikoff B (2005) Towards the rational development of molecularly imprinted polymers: ${ }^{1} \mathrm{H}$ NMR studies on hydrophobicity and ion-pair interactions as driving forces for selectivity. Biosens Bioelectron 20:1884-1893

16. Schmaljohann D, Beyerlein D, Nitschke M, Werner C (2004) Thermo-reversible swelling of thin hydrogel films immobilized by low-pressure plasma. Langmuir 20:10107-10114

17. Malosse L, Palmas P, Buvat P, Adès D, Siove A (2008) Novel stoichiometric, noncovalent pinacolyl methylphosphonate imprinted polymers: a rational design by NMR spectroscopy. Macromolecules 41:7834-7842 